cathode drop are quite high, namely.

$$
\begin{aligned}
\varepsilon_{+\nu} & =\sum_{\mu=2}^{v} e\left(U_{\mathrm{i} \mu}-\varphi\right)+e U_{\mathrm{c}}, \\
\varepsilon_{+2} & =34[\mathrm{eV}] ; \varepsilon_{+3}=66[\mathrm{eV}] ; \varepsilon_{+4}=120[\mathrm{eV}] .
\end{aligned}
$$

Even though we have no detailed information about the accommodation coefficients of these processes these energies offer a basis for an explanation of the

1 G. Ecker, Betr. a. d. Plasmaphysik II, 406 [1971].

2 G. Ecker, General Electric TIS Report 71-C-195 [1971], Schenectady, New York.

3 G. Ecker, Erg. exakt. Naturwiss. 33, 1 [1961].

4 G. ECKer, Z. Physik 136, 556 [1954].

5 I. G. Kesaev, Cathode Processes in the Mercury Arc, State Power Engineering Press, Moscow 1961.

6 G. Ecker, Z. Naturforsch. 25 a, 935 [1971].

7 G. Ecker, Klassische Probleme der Gaselektronik in moderner Sicht, Rheinisch-Westfälisehe Akademie der Wissenschaften, Vorträge 1972, Westdeutscher Verlag, Opladen. surprising phenomenon that ions have been observed emerging from the cathode spot area with energies usually between $20-60[\mathrm{eV}]$, i. e. with energies well above the total typical vacuum voltage ${ }^{14}$.

Further study of this question, in particular the consideration of the mechanism by which the ions escape the spot area without loosing their energy, is in progress.

${ }^{8}$ E. L. Murphy and R. H. Good, Jr., Phys. Rev. 102, 1464 [1956].

9 M. H. Kogan and H. K. Makaschew, J. Mechanics of Liquids and Gases 6, 3 [1971].

10 G. Ecker, Z. Physik 135, 105 [1953].

11 M. Steenbeck, Wiss. Veröffentl. Siemens Concern XIX (1940), 59

12 Th. Peters, Z. Phys. 144, 612 [1956].

13 M. Steenbeck, Plasma Physik 1, 1 [1960/61].

14 W. D. Davis and H. C. Miller, J. Appl. Phys. 40, 2212 [1969].

\title{
The Non-Stationary Metal Vapour Arc
}

\author{
G. ECKER
}

Institut für Theoretische Physik, Ruhr-Universität Bochum

(Z. Naturforsch. 28 a, 428-437 [1973] ; received 7 December 1972)

This investigation studies the effect which the motion of the cathode spot has on its E-diagram. The motion is depicted as a sequence of steps of a finite residence time.

The spot motion affects essentially only the energy characteristics $T_{\mathrm{e}}$ which in comparison to the stationary characteristics $T_{\mathrm{es}}$ are shifted to smaller values. Hereby the critical currents $I_{0}, I_{1}$ are raised in comparison to the corresponding stationary limits $I_{0 \mathrm{~s}}, I_{1 \mathrm{~s}}$.

Particularly attractive are the phenomena found in connection with the dependence of the spot velocity $v$ on the spot current $I$. If the spot velocity increases with the spot current stronger than $v \propto I^{1 / 2}$ then the E-diagram reveals the existence of an upper limit $I_{u}$ for the spot current. This result can be used to explain qualitatively the experimentally observed phenomena of "spot multiplicity" and "spot extinction".

Quantitative conclusions are obstructed by the lack of knowledge about the velocity dependence on the spot current, $v(I)$. Experimental and theoretical studies to provide a better understanding of the physical background and the analytical laws describing the motion of the cathode spots are urgently needed.

\section{Introduction}

In an earlier investigation of the metal vapour $\operatorname{arc}^{1}$ we considered the stationary cathode spot (SCS). Using the E-diagram technique ${ }^{2,3}$ we studied characteristics of charge conservation, particle conservation and energy conservation. Apart from a relatively close limitation of the current densities and the temperatures in the cathode spot we observed the occurrence of two different spot-modes $(0,1)$.

Reprint requests to Prof. Dr. G. Ecker, Theoretische Physik, Lehrstuhl I, Ruhr-Universität Bochum, D-4630 Bochum-Querenburg, Buscheystraße, Gebäude NB 7/152.

We further found a requirement for a minimum spot current $\left(I_{0}, I_{1}\right)$ for the stationary operation of a cathodic onset in these modes.

In the consecutive treatment of the metal vapour or vacuum $\operatorname{arc}^{4}$ we improved the understanding of the cathode spot mechanism by including simultaneously the analysis of the sheath and the space charge region and by taking into account multiply charged ions. In this way we found the variation of the electron temperature $T_{\text {- }}$ and the cathode drop $U_{\mathrm{c}}$ with the spot surface temperature $T$ and the total current density $j$.

In principal these new results ask for a recalculation of the E-areas derived in ${ }^{1}$ utilizing the more accurate data of ${ }^{4}$. This analysis is in progress but 
quite cumbersome. However, as was already argued in ${ }^{4}$, it can be anticipated that this recalculation will approximately reproduce the E-areas of ${ }^{1}$ and yield changes only in the rest of the $(j-T)$-diagram which is not directly relevant for the physical consequences studied here.

It seems noteworthy that neither the original investigation in ${ }^{1}$ nor the improvement in ${ }^{4}$ yield an upper limit for the total spot current which might be expected from the experimental evidence of the phenomenon of spot multiplicity. This leads us to suspect that our model is not complete yet.

In fact the stationary cathode spot presents an idealization of the true spot in that the true spot is practically always in motion. This non-stationarity may - as we already suspected in ${ }^{1}$ - reduce the E-area, alter the critical minimum currents $I_{\text {cr }}$ or even yield the expected upper limitation for $I$. Such influences might also have a bearing on the conclusions drawn for the chopping and stability qualities of the vacuum $\operatorname{arc}^{5}$.

\section{Principal Considerations of the Non-Stationary E-Diagram}

As far as the basic features of this investigation are concerned, we refer the reader to the References ${ }^{1-3}$.

As in 1,6 we limit the existence area through "unidirected approximations", so called characteristics. Each of these characteristics yields a $T(j)$. dependence because it neglects one or a combination of the laws describing the spot mechanism. According to the large number of possible neglections, we have a large choice of possible characteristics.

We select here the same dependencies as in the treatment of the stationary spot where we restricted ourselves to three such characteristics and termed them for the reason of their physical background:

characteristic of charge conservation (c), characteristic of neutral particle conservation (n), characteristic of energy conservation (e).

Of course, under these circumstances it is advised to orientate our analysis of the nonstationary cathode spot (NCS) closely at the procedure used in ${ }^{1}$. In fact we will simply start with the equations of ${ }^{1}$ and discuss to what extent alterations are required due to the non-stationarity:

We consider an arc-spot which moves with a certain constant velocity across the surface of the ca- thode. The motion is interpreted on the basis of the idea that the seemigly continuous motion of the spot is actually a sequence of steps in the following sense. The arc operates in one place for a certain time $\tau_{0}$, then discontinuously steps to a neighbouring site displaced about one spot diameter $(2 a)$ and here operates again for a time $\tau_{0}$, which we call the residence time. This "step motion" is supported by experimental evidence.

It is clear that within the frame of this model the relation

$$
\tau_{0}=O(a / v)=O\left(I / j v^{2}\right)^{1 / 2}
$$

holds, where $(a)$ denotes the radius of the spot and (v) the spot velocity.

This relation (1) allows an estimate of the time $\tau_{0}$ which characterizes the non-stationarity and which will prove useful in discussing the extent of applicability of the SCS-theory to the NCS-analysis.

We know from experiment that for a Cu-Cathode the spot currents are in the range $I=O\left(10^{2}\right)$ [A] and their spot velocities in the range $v \leqq O\left(10^{4}\right)$ $[\mathrm{cm} / \mathrm{sec}]$. Further it follows from the E-diagram in ${ }^{1}$ that the current densities are $j=O\left(10^{6}\right)\left[\mathrm{A} / \mathrm{cm}^{2}\right]$. Therefore

$$
\tau_{0} \geqq O\left(10^{-6}\right)[\mathrm{s}]
$$

holds for the residence time of the spot.

Knowing $\tau_{0}$ we now decide whether the laws describing the characteristics of charge conservation (c), neutral particle conservation (n) and energy conservation (e) relax to stationarity in a time $\tau_{\mathrm{r}} \ll \tau_{0}$ or whether they require non-stationary adjustments.

\section{Calculation of the Non-Stationary Characteristics}

As stated, we orientate our calculations at the corresponding relations of the SCS-theory.

\section{Characteristic of Charge Conservation}

We use the equations presented in Ref. ${ }^{1}$ in the form

$$
\begin{gathered}
j_{\mathrm{e}}=e \int_{\varepsilon_{\mathrm{m}}}^{\infty} D\left(E_{\mathrm{c}}, \bar{\varepsilon}\right) N(\bar{\varepsilon}, T, \varphi) \mathrm{d} \bar{\varepsilon} \\
D\left(E_{\mathrm{c}}, \bar{\varepsilon}\right)=\left[1+\exp \left(-\frac{2 i}{\hbar} \int_{x_{1}}^{x^{2}} p(x) \mathrm{d} x\right)\right]^{-1} \\
N(\bar{\varepsilon}, T, \varphi)= \\
\quad\left[4 \pi k T m_{-} / h^{3}\right] \ln [1+\exp \{-(\bar{\varepsilon}-\varphi) / k T\}], \\
E_{\mathrm{c}}{ }^{2}=4 / \varepsilon_{0}\left(m_{+} U_{\mathrm{c}} / 2 e\right)\left\{j_{+}\left(m_{+} / m_{-}\right)^{1 / 2}-j_{-}\right\}
\end{gathered}
$$




$$
\begin{gathered}
j=j_{+}+j_{\mathrm{e}}\left(E_{\mathrm{c}} T\right), \\
j_{+} / j \leqq U_{\mathrm{c}} /\left(U_{\mathrm{c}}+U_{\mathrm{i}}\right) .
\end{gathered}
$$

Here $j_{\mathrm{e}}$ and $j_{+}$designate respectively the absolute value (a.v.) of the electron emission density from and the ion current density to the cathode. $D$ is the emission probability, $N$ the supply function. $\bar{\varepsilon}$ designates the electron energy, $p$ the momentum perpendicular to the electrode surface. $x_{1}$ and $x_{2}$ are defined through $p^{2}(x)=0 . \varphi$ is the work function of the cathode material. $E_{\mathrm{c}}$ designates the a. v. of the electric field at the cathode surface. $U_{\mathrm{c}}$ is the cathode drop and $U_{\mathrm{i}}$ the ionization potential. The $(<)$-sign of Eq. (8) admits the existence of the spot in the range

$$
T>T_{\mathrm{c} 1}(j)
$$

where the characteristic $T_{\mathrm{c} 1}(j)$ is the solution of Eqs. (3) - (8) using the (=)-sign in Equation (8).

The applicability of these relations $(3)-(8)$ to the non-stationary moving spot can be judged by comparing the relevant relaxation times with the residence time $\tau_{0}$.

The fastest process of the phenomena described in Eqs. $(3)-(8)$ is the relaxation of the $(T-F)$ emission formulated in Eqs. (3) - (5). Its order of magnitude is characterized by

$$
\tau_{\mathrm{c} 1}=l_{\mathrm{m}} / \bar{v}_{\mathrm{em}}=O\left(10^{-14}\right)[\mathrm{s}]
$$

where $l_{\mathrm{m}}$ is the characteristic length of the metal structure and $\bar{v}_{\mathrm{em}}$ the average velocity of the electrons within the metal. Obviously $\tau_{\mathrm{c} 1}$ is many orders of magnitude smaller than $\tau_{0}$ and consequently we treat the emission process quasi-stationary.

Next we consider Eq. (6) and characterize the corresponding relaxation time by

$$
\begin{aligned}
\tau_{\mathrm{c} 2} \leqq l_{\mathrm{sp}} / v_{+\mathrm{s}} \cong 10\left(k T_{-} / \varepsilon_{\mathrm{s}}\right)^{1 / 2} \omega_{\mathrm{p}+}{ }^{-1} \approx \omega_{\mathrm{p}+}{ }^{-1} \\
\leqq O\left(10^{-8}\right)[\mathrm{s}]
\end{aligned}
$$

where $l_{\mathrm{sp}}$ designates the extension of the space charge region, $v_{+s}$ the average velocity of the ions ini the space charge region, $\varepsilon_{\mathrm{s}}$ the corresponding energy and $\omega_{\mathrm{p}}$ t the plasma ion frequency. Here we used the result from ${ }^{4}$ that $l_{\mathrm{sp}}=O\left(10 \lambda_{-\mathrm{D}}\right)$ holds.

Obviously $\tau_{\mathrm{c} 2}$ is also much smaller than $\tau_{0}$ so that we may use Eq. (6) unchanged.

The current continuity of Eq. (7) too is characterized in its relaxation by the quantity $\tau_{\mathrm{c} 2}$. We can therefore use Eq. (7) for our moving spot.

Finally in judging Eq. (8) we remember that its background is the analysis of the ionization region.
Due to the assumption of quasi equilibrium the redistribution of this plasma caused by the moving spot is governed by ionization and recombination processes and we therefore have the relataxion time

$$
\tau_{\mathrm{c} 3}=l_{\mathrm{p} 1} / \bar{v}_{-} \leqq O\left(10^{-9}\right)[\mathrm{s}]
$$

where $l_{\mathrm{pl}}$ characterizes an average mean-free-path

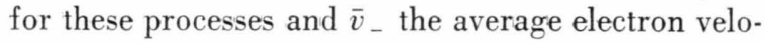
city in the plasma. Here we have taken into account that the pressure of the neutral gas cloud is always near or above one atmosphere.

Conclusion: Since we have $\left(\tau_{\mathrm{c} 1}, \tau_{\mathrm{c} 2}, \tau_{\mathrm{c} 3}\right) \ll \tau_{0}$ the characteristic of charge conservation as calculated in the SCS-theory can be transferred to the Ediagram of the NCS-investigation.

\section{Characteristic of Neutral Particle Conservation}

In the SCS-treatment we considered two necessary conditions for neutral particle conservation corresponding to the limits that all or none of the neutral particles vapourized are ionized in the plasma cloud.

They read

$$
j_{+}\left(j, T_{\mathrm{n} 1}\right)=\frac{e}{4} \frac{p_{\mathrm{ev}}\left(T_{\mathrm{n} 1}\right)}{\left(m_{+} k T_{\mathrm{n} 1} / 3\right)^{1 / 2}}
$$

the other

$$
j=\left(\frac{I Q^{2} \mathrm{ei}}{\alpha_{\mathrm{n}}{ }^{2}}\right) \frac{p^{2}{ }_{\mathrm{ev}}\left(T_{\mathrm{n} 2}\right)}{\left(k T_{\mathrm{n} 2}\right)^{2} \pi}
$$

where $p_{\mathrm{ev}}$ denotes the equilibrium vapour pressure, $Q_{\mathrm{ei}}$ the cross-section for ionizing electron-neutral collisions, $I$ the total spot current and $\alpha_{\mathrm{n}}$ an uncertainty factor $\left(\alpha_{n}>1\right)$. Equations (13), (14) together with Eqs. (3) - (7) provide two sets of characteristics $T_{\mathrm{n} 1}, T_{\mathrm{n} 2}$ requiring

$$
T \geqq T_{\mathrm{n} 1}(j), \quad T_{\mathrm{n} 2}(j) .
$$

Having secured the quasi-stationary treatment of Eqs. (3) - (7) in the previous section the new relaxation time to be considered is the time $\tau_{\mathrm{n}}$ of the adjustment of the neutral vapour density to a given surface temperature. This time $\tau_{\mathrm{n}}$ is characterized by the average collision time of neutral cloud particles with the electrode surface

$$
\tau_{\mathrm{n}}=l_{\mathrm{n}} / \bar{v}_{\mathrm{n}},
$$

where $l_{\mathrm{n}}$ describes the mean-free-path and $\bar{v}_{\mathrm{n}}$ the average velocity of the neutrals in front of the cathode spot surface. Since all mean-free-paths - even 
that of the electrons - are assumed to be small in comparison to the spot extension (a) and $\bar{v}_{\mathrm{n}} \gg v$ holds, it is clear that we have $\tau_{\mathrm{n}} \ll \tau_{0}$.
Consequence: We can use in the E-diagram of the movinig spot (NCS) the $T_{\mathrm{n}}$-characteristics of the (SCS) -analysis.

\section{Characteristic of Energy Conservation}

The stationary energy characteristic was described by the relation

$$
j\left\{\left(U_{\mathrm{c}}+U_{\mathrm{i}}\right) \underset{j}{j_{+}\left(j, T_{\mathrm{e}}\right)}-\left(\varphi-\frac{0.32}{V \pi} I^{1 / 2} j^{1 / 2} \varrho\left(T_{\mathrm{e}}\right)\right)\right\}=\frac{\varepsilon}{4} \frac{p_{\mathrm{eV}}\left(T_{\mathrm{e}}\right)}{\left(m_{+} k T_{\mathrm{e}} / 3\right)^{1 / 2}}+T_{\mathrm{e}} \cdot w\left(T_{\mathrm{e}}\right)\left(\frac{j \pi}{I}\right)^{1 / 2}
$$

where the existence area is limited by

$$
T<T_{e}(j, I) \text {. }
$$

In this relation $\varrho$ and $w$ denote respectively the specific resistivity and the heat conductivity of the cathode material. The relaxations of all processes entering into Eq. (16) where covered in the previous discussion of charge conservation and neutral particle conservation, - except one:

The relaxation time $\tau_{\mathrm{e}}$ of the temperature distribution within the cathode.

To estimate this relaxation time $\tau_{\text {e }}$ consider a semi-infinite medium with $w(T)$ at an initial temperature $T_{0}$ (room temperature) with a plane surface to which a constant energy density flux $J$ is applied over a circular area of radius $(a)$. The time development of the temperature distribution in the medium can than be described ${ }^{7}$ by

$$
T=\frac{a J}{2 w} \int_{0}^{\infty} J_{0}(\lambda r) J_{1}(\lambda a)\left\{e^{-\lambda z} \operatorname{erfc}\left[\frac{z}{2(\delta t)^{1 / 2}}-\lambda(\delta t)^{1 / 2}\right]-e^{\lambda z} \operatorname{erfc}\left[\frac{z}{2(\delta t)^{1 / 2}}-\lambda(\delta t)^{1 / 2}\right]\right\} \frac{\mathrm{d} \lambda}{\lambda}
$$

where $\delta$ designates the thermal diffusivity

$$
\delta=w / \varkappa=w / c d
$$

$d$ is the density and $\varkappa, c$ describe the heat capacity per unit volume respectively unit mass. $z$ is the coordinate perpendicular to the surface in the center of the circle, $r$ the corresponding radial coordinate. $J_{s}$ denotes the Bessel function of order $s$.

We can restrict our considerations to the $z$-axis $(r=0)$ since for large distances the problem has spherical symmetry and so the $z$-axis is characteristic whereas for small distances the problem can be considered plane and our results hold across the whole (a)-area.

For $r=0$ Eq. (19) reduces to

$$
T=\frac{2 J}{w}(\delta t)^{1 / 2} \quad\left\{\operatorname{ierfc}\left(\frac{z^{2}}{4 \delta t}\right)^{1 / 2}-\operatorname{ierfc}\left(\frac{z^{2}+a^{2}}{4 \delta t}\right)^{1 / 2}\right.
$$

where ierfc is the integral of the error function complementary.

We are solely interested in the surface temperature at $z=0$ and for that point Eq. (21) shows by means of a power expansion that the relaxation time to the stationary value is given by

$$
\tau_{\mathrm{e}}=a^{2} / \delta .
$$

For a number of typical examples the relaxation times $\tau_{\mathrm{e}}$ are calculated from the knowledge of the diffusivity and the spot size from the E-diagram.

\begin{tabular}{llllll} 
& $\mathrm{Fe}$ & $\mathrm{Cu}$ & $\mathrm{Mo}$ & $\mathrm{W}$ & $\mathrm{Cd}$ \\
\hline$O\left(\tau_{\mathrm{e}}\right)[\mathrm{s}]$ & $10^{-4}$ & $10^{-5}$ & $10^{-6}$ & $5 \cdot 10^{-7}$ & $10^{-8}$
\end{tabular}

These numbers show that in general the condition $\tau_{\mathrm{e}} \ll \tau_{0}$ is not fulfilled so that we have to solve our energy conservation characteristic non-stationary.

\section{Non-Stationary Energy Characteristic}

As we know from the solution of the stationary problem the net-influx $J$ to the spot area on the cathode surface is a function of the instantaneous cathode spot temperature. This temperature varies with time. Consequently $J$ is an implicit function of time, $J[T(t)]$. Therefore it is our problem to solve the equation of heat conduction in the cathode

$$
\partial \varkappa(T) T(\boldsymbol{x}, t) / \partial t=\boldsymbol{\nabla}\{w(T) \nabla[T(\boldsymbol{x}, t)]\}
$$

subject to the boundary condition of a temperature dependent energy influx $J(T)$ in a circular area at the surface of a semi-infinite medium bounded by a planar surface. This provides us with an integro differential equation for the temperature if we use 
the approach of Green's function. We neither know of any analytical solution of this problem ${ }^{8}$ nor do we consider this as a task which we should tackle in this paper.

Instead we recall that for the E-diagram, it is sufficient to calculate a value $T_{\mathrm{e}}$ larger tan the true temperature $T$ in the surface spot area

$$
T_{\mathrm{e}}>T \text {. }
$$

In this spirit let us then calculate the surface temperature of a model where the energy supply density $J[T(t)]$ is extended from the actual circular area (a) to the whole surface plane of the semispace. The temperature $T_{\mathrm{e}}$ calculated in this way will certainly satisfy the relation (25). Moreover, the temperature $T_{\mathrm{e}}$ will even be a close approximation to $T$ as long as the diffusion length $(\delta t)^{1 / 2}$ is smaller than the radius of the spot $(a)$. This holds in the whole range of our interest since for a time $t=\left(a^{2} / \delta\right)^{1 / 2}$ we approach the stationary state. The assumption of a plane energy supply is therefore a good approximation for our calculation and completely save in the sense of Equation (25).

Still no general analytical solution can be found due to the temperature dependence of $w$ and $\%$. Again we may take advantage of the E-diagram technique by choosing $w$ and $x$ constant, but such that the temperature $T_{\mathrm{e}}$ is increased in the direction of Equation (25).

With $w$ and $\varkappa$ constant and a time dependent plane energy influx $J(t)$ the analytical solution is ${ }^{11}$

$$
T(z, t)=\frac{1}{2\left(\pi w_{0} \varkappa_{0}\right)^{1 / 2}} \int_{0}^{t} \frac{e^{-z^{2} / 4 \delta\left(t-t^{\prime}\right) J\left(t^{\prime}\right)}}{\left(t-t^{\prime}\right)^{1 / 2}} \mathrm{~d} t^{\prime}+T_{0}
$$

where $w_{0}$ and $\varkappa_{0}$ denote the constant values as specified above. Since we are interested in the surface temperature only, Eq. (26) simplifies to equation

$$
T_{\mathrm{e}}(t)=\frac{1}{2\left(\pi w_{0} \varkappa_{0}\right)^{1 / 2}} \int_{0}^{t} \frac{J\left[T_{\mathrm{e}}\left(t^{\prime}\right)\right]}{\left(t-t^{\prime}\right)^{1 / 2}} \mathrm{~d} t^{\prime}+T_{0}
$$

which is an integral equation for the function $T_{\mathrm{e}}(t)$. A very awkward integral equation since the energy influx according to Eq. (17) is given by

$$
\begin{array}{r}
J\left(j, I, T_{\mathrm{e}}(t)\right)=j\left\{\left(U_{\mathrm{c}}+U_{\mathrm{i}}\right) \frac{j_{+}\left(j, T_{\mathrm{e}}\right)}{j}\right. \\
\left.-\left(\varphi-\frac{0.32}{V \pi} I^{1 / 2} j^{1 / 2} \varrho\left(T_{\mathrm{e}}\right)\right)\right\}-\frac{\varepsilon}{4} \frac{p_{\mathrm{ev}}\left(T_{\mathrm{e}}\right)}{\left(m_{+} k T_{\mathrm{e}} / 3\right)^{1 / 2}}
\end{array}
$$

where $j_{+}\left(j, T_{\mathrm{e}}\right)$ must be calculated from Equations (3) $-(7)$.
Nevertheless it is possible to solve Eqs. (27), (28), (3) - (7) numerically by an iterative procedure and with that to find the characteristic $T_{\mathrm{e}}(j, I, t)$ as a function of time having for $t=0$ the value $T_{\mathrm{e}}=T$ and approaching for $t \rightarrow \infty$ the stationary solutions $T_{\text {es }}$.

With that we know all three characteristics as a function of time and can therefore calculate the Earea as a function of time.

\section{The Non-Stationary E-Area}

From the relation (27) and the structure of the SCS-E-Diagram it is quite obvious that for $t=0$ no self-sustained operation of the arc is possible. This means that immediately after a displacement - after a step - the spot cannot operate self-sustained. But during this initial phase it may still profit from the remains of the operation in the preceding step and consequently one can argue that self-sustainement is not required yet. On the other hand the moving arc certainly cannot operate continuously if it does not reach self-sustainement at any stage during its residence time $\tau_{0}$. Consequently, for the continuous operation of a moving spot it is a necessary requirement:

A finite E-Area must be available in the nonstationary E-Diagram at least at the time $t=\tau_{0}$.

We observe htat the restriction to a necessary requirement is again in the spirit of the E-diagram technique.

To evaluate the consequences of this necessary requirement, we plot the stationary characteristics $T_{\mathrm{c}}(j)=T_{\mathrm{cs}}(j)$ and $T_{\mathrm{n}}(j)=T_{\mathrm{ns}}(j)$ together with the non-stationary characteristics $T_{\mathrm{e}}\left(j, I, \tau_{0}\right)<T_{\mathrm{es}}(j, I)$ into a diagram ${ }^{12}$. Due to the dependence of $\tau_{0}$ on the spot velocity $v$ [see Eq. (1)] the characteristics $T_{\mathrm{e}}$ must be expected to depend also on the velocity $v$, $T_{e}(j, I, v)$.

The characteristics $T_{\mathrm{c}}=T_{\mathrm{cs}}$ an $T_{\mathrm{n}}=T_{\mathrm{ns}}$ are shown in Figures 1 and 2.

The evaluation of the $T_{\mathrm{e}}$ characteristics requires the specification of the spot velocity $v$ as a function of the spot current $I$.

The derivation of this relation $v(I)$ as a function of the experimental conditions cannot be subject of this paper. We should take this information either from experimental evidence or from earlier theoretical attempts. There is such experimental evidence 


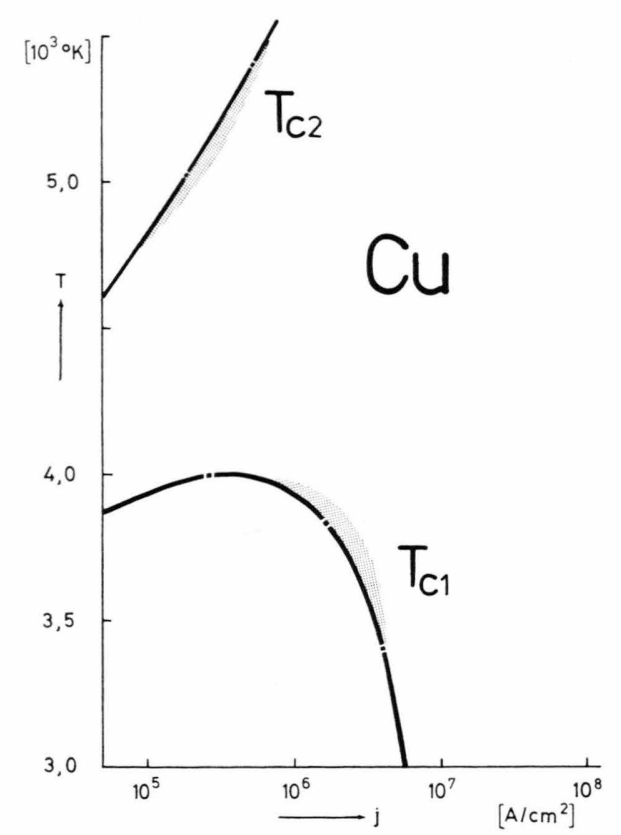

Fig. 1

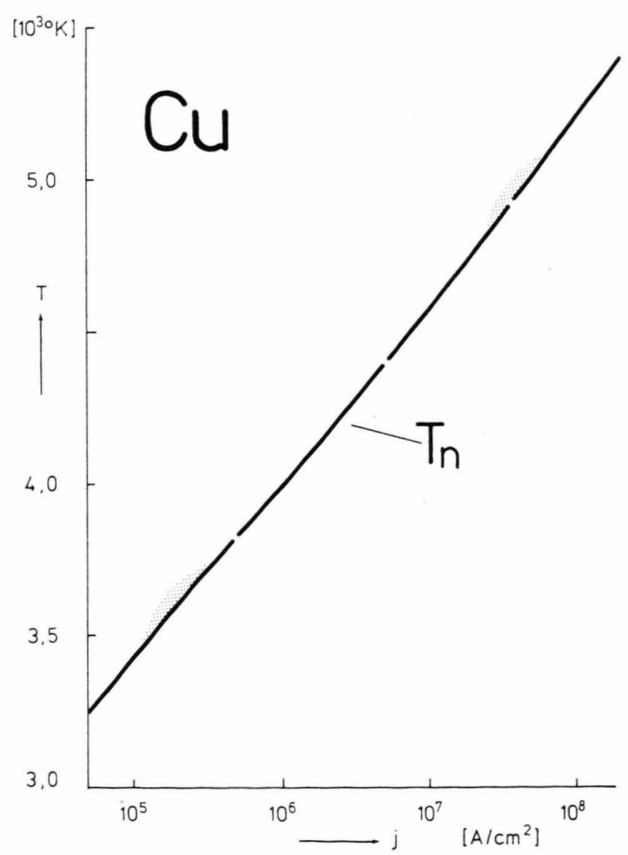

Fig. 2

Figs. 1 and 2. Characteristics of charge and neutral particle conservation $T_{\mathrm{c}}, T_{\mathrm{n}}$ identical with the corresponding stationary characteristics $T_{\mathrm{cs}}, T_{\mathrm{ns}}$ calculated in ${ }^{1}$ of this investigation of the vacuum arc.

(Refs. ${ }^{13-20}$ ) but it is rather limited and not in the range of parameters where we would want it. There is an abundance of theoretical endeavours ${ }^{21-29}$ but most of them offer only a vague physical concept without analytical or even quantitative evaluation. Moreover, recent emotional dialectics contributed only to the general feeling of insecurity in this field ${ }^{30}$.

In this situation we consider it reasonable not to use uncertain values of experimental or theoretical origin but rather to study the consequences of the spot motion for various cases of characteristic dependencies $v(I)$.

The velocity dependence on $I$ is important since it influences the $T_{\mathrm{e}}$-characteristics via the upper limit of integration in

$T_{\mathrm{e}}(j)=T_{\mathrm{e}}\left(j, \tau_{0}\right)=\frac{1}{2\left(\pi w_{0} \varkappa_{0}\right)^{1 / 2}} \int_{0}^{\tau_{0}} \frac{J\left[T_{\mathrm{e}}\left(t^{\prime}\right)\right]}{\left(t-t^{\prime}\right)^{1 / 2}} \mathrm{~d} t^{\prime}+T_{0}$

where $\tau_{0}=\left(I / j v^{2}\right)^{1 / 2}$ holds.

It is obvious that we should distinguish two essentially different cases $(1,2)$.

$$
\begin{array}{ll}
v=c \cdot I^{v} ; & v \leqq 1 / 2 ; \\
v=c \cdot I^{v} ; & v>1 / 2 .
\end{array}
$$

Case 1: As can be seen from Eq. (1) $\tau_{0}$ increases or stays constant with increasing current. Under these circumstances it folows from the structure of Eq. (29) that the non-stationary $T_{\mathrm{e}}(j, I)$ characteristics in their dependence on $I$ form a set of curves shifting with increasing $I$ monotonously to larger values of $T$. Of course the relation $T_{e}(j, I)$ $<T_{\mathrm{es}}(j, I)$ always holds.

C a s e 2 : Again $T_{\mathrm{e}}(j, I)<T_{\mathrm{es}}(j, I)$ holds. However, the decrease of $\tau_{0}$ with increasing $I$ now causes an important change. For small values of $I$ the $T_{\mathrm{e}^{-}}$ curves shift with increasing $I$ to larger values of $T$ as in case 1 and as in the stationary case. However, above a certain current $I$ the characteristics start to move down again due to the decrease in $\tau_{0}$. As we will see, this effect has decisive consequences with respect to the E-area.

As a typical example for the structure of case 1 , Fig. 3 demonstrates the $T_{\mathrm{e}}$-characteristics for a constant velocity $(c=10[\mathrm{~m} / \mathrm{s}], v=0)$. For the purpose of comparison Fig. 4 presents the corresponding $T_{\text {es }}$ curves.

The principal features of case 2 are shown in Fig. 5 where we have chosen $(c=1 / 32[\mathrm{~m} / \mathrm{s} \mathrm{A}]$, $v=1)$. The figures clearly demonstrate the predicted rise and fall of the characteristics with increasing total current $I$. 


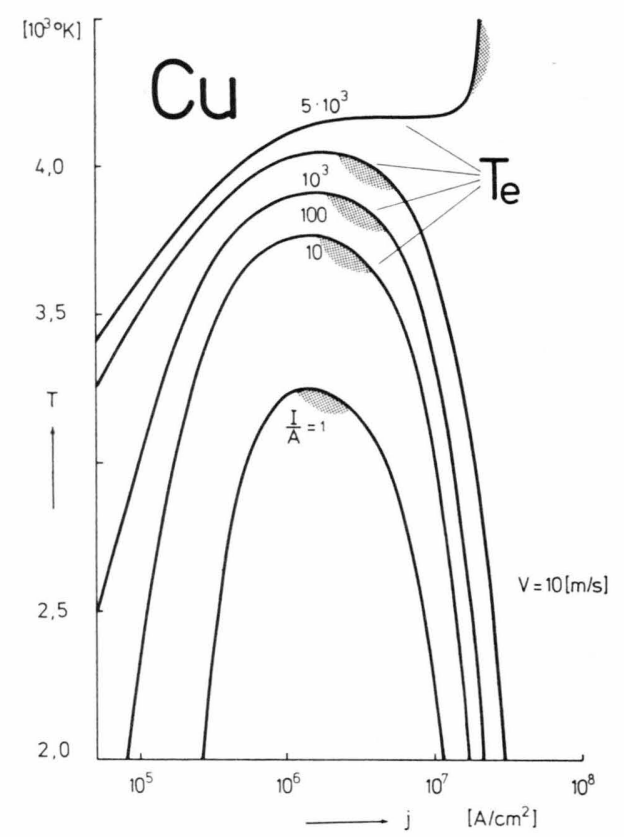

Fig. 3

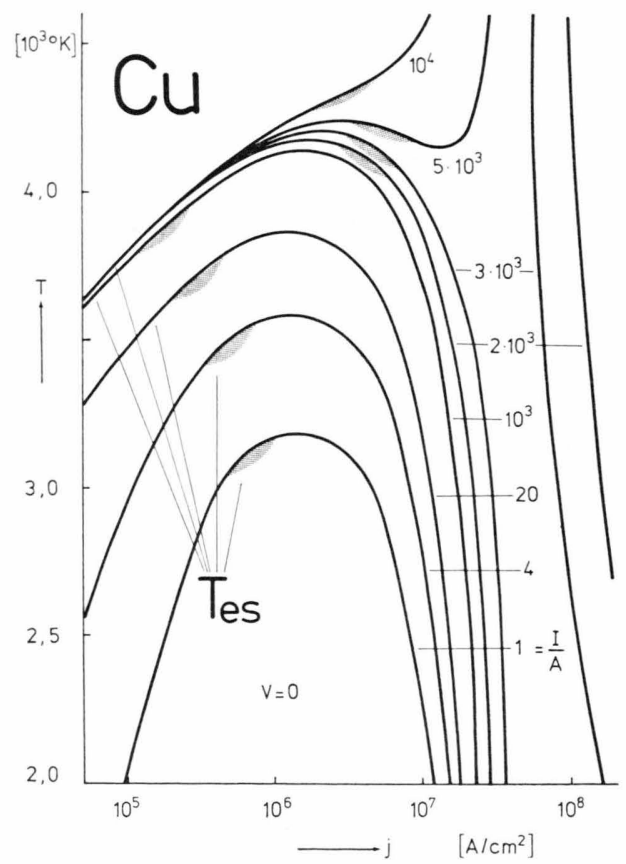

Fig. 4

Figs. 3 and 4. Non-stationary energy characteristics $T_{\mathrm{e}}$ for a spot velocity $v=10[\mathrm{~m} / \mathrm{s}]$ and stationary spot characteristics $T_{\mathrm{es}}$ as calculated in ${ }^{1}$.

Noteworthy is the fact that the (1)-mode is practically precluded in both cases. In case 1 this mode disappears for all currents below the unphysically
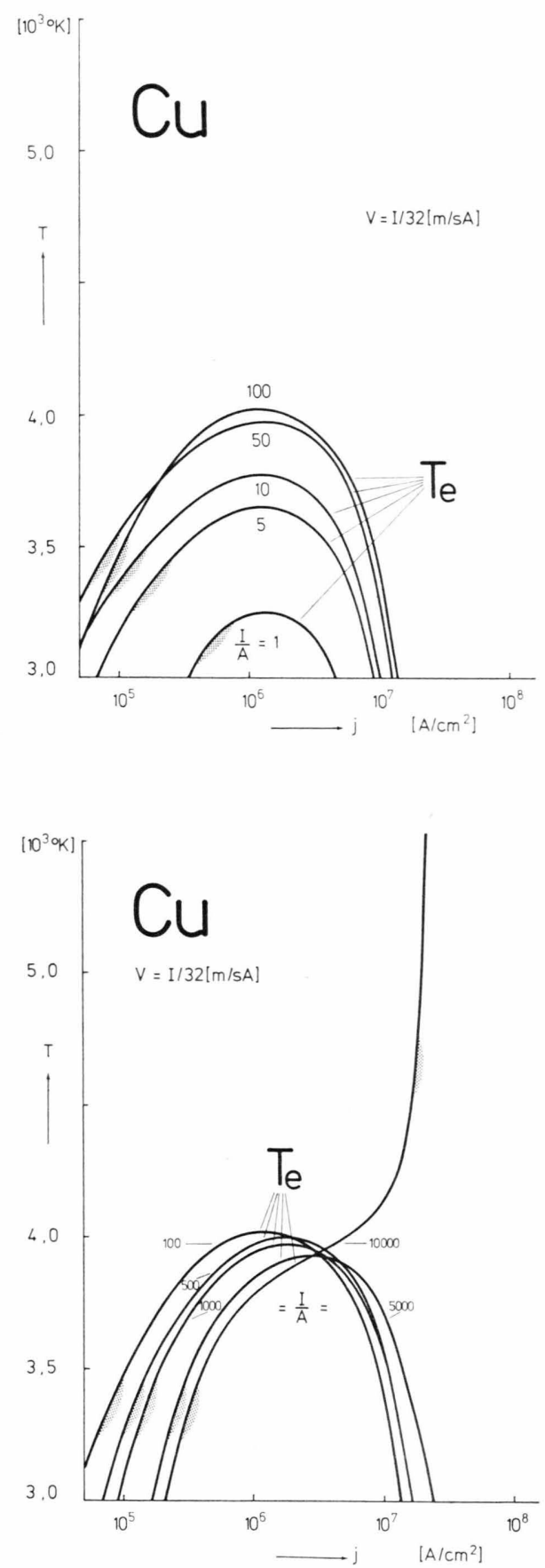

Fig. 5. Rising and falling non-stationary energy characteristics $T_{\mathrm{e}}$ for a spot velocity $v$ depending on the spot current $I$ according to the relation $v=I / 32[\mathrm{~m} / \mathrm{s} \mathrm{A}]$.

high limit of about 5000 A. In case 2 it disappears altogether ${ }^{31}$.

The construction of the existence area for an example of case 2 results in Figure 6. For reasons of 


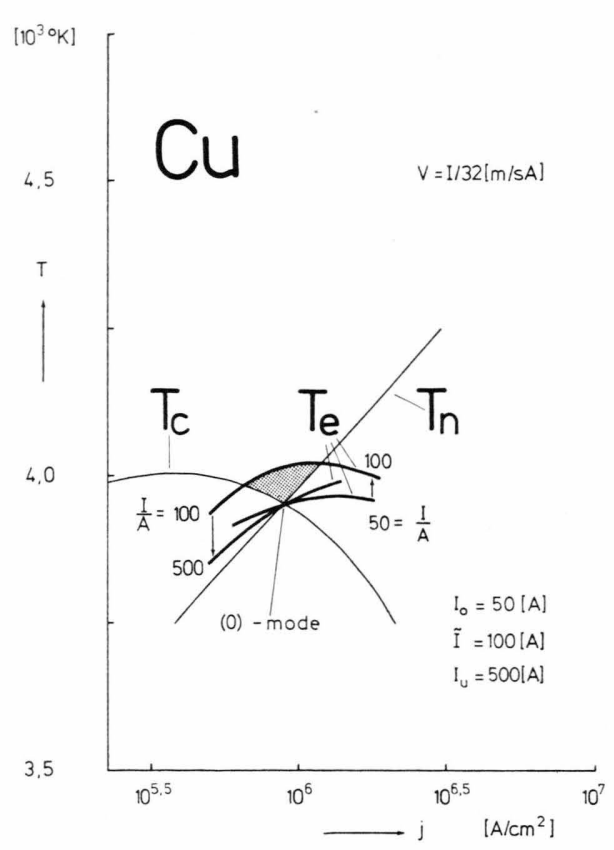

Fig. 6. Structure of the E-area of a cathode spot moving with the velocity $v=I / 32[\mathrm{~m} / \mathrm{s} \mathrm{A}]$. The arrows indicate the shift of the $T_{\mathrm{e}}$-characteristics with increasing current $I . I_{0}$ designates the minimum current required for existence, $\tilde{I}$ the current at which the shift of the $T_{\mathrm{e}}$-characteristics changes its sign, $I_{\mathrm{u}}$ the maximum current for existence.

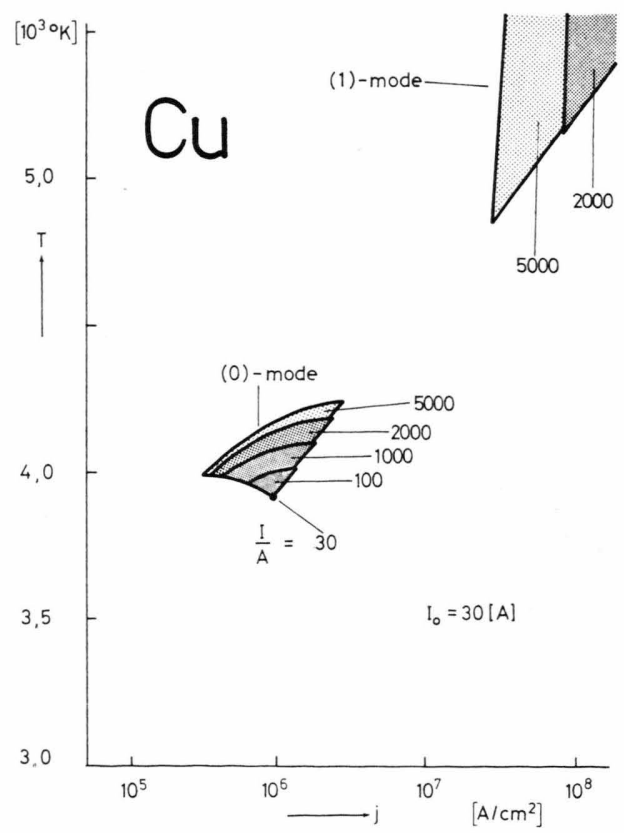

Fig. 7. Existence diagram of the stationary spot as calculated in ${ }^{1} . I_{0}$ is the minimum current required for the existence of a spot at rest. comparison Fig. 7 shows the corresponding stationary existence area.

We note that the critical minimum current $I_{0}$ below which we expect fluctuations and extinction is in the non-stationary case larger than in the stationary case, - as expected. Most important however, the continuous increase of the existence area observed in the stationary diagram is not recovered in the non-stationary diagram of case 2 . In fact the existence area in the non-stationary case first increases with increasing current and then passing a certain current $\tilde{I}$ decreases reaching again zero at a critical upper current $I_{\mathrm{u}}$. Spot velocities in the range of case 2 produce an upper limitation $I_{\mathrm{u}}$ for the spot current.

Since these limiting currents $I_{0}, I_{\mathrm{u}}$ are of central interest, we calculated their values as a function of the parameter $c$ for $v=1$.

The result is shown in Figure 8. It shows that the upper and lower current limit approach each other with increasing velocity. When they intersect we have reached a veocity where spot existence is excluded altogether.

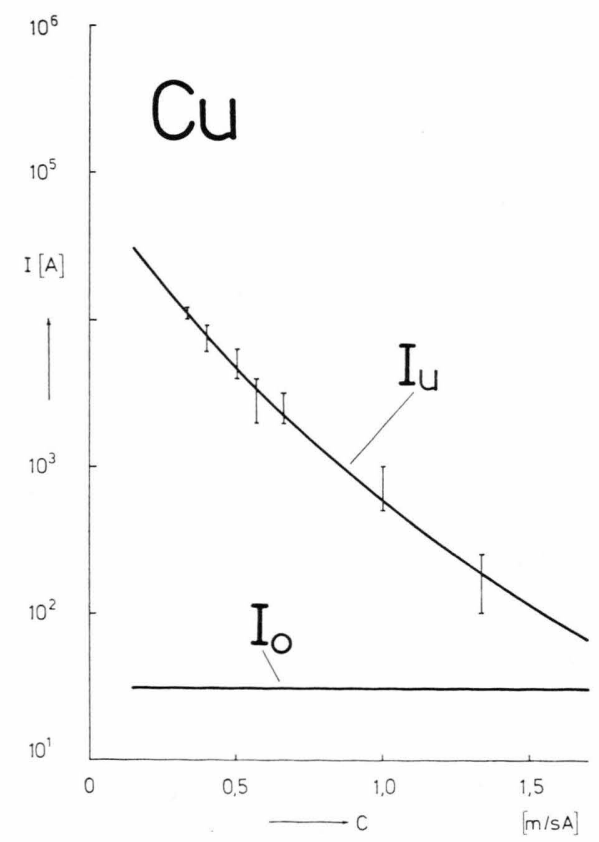

Fig. 8. Maximum and minimum critical current $I_{\mathrm{u}}, I_{0}$ required for the existence of a spot moving according to the relation $v=c I$ as a function of the constant $c$. 


\section{Summary and Discussion}

In this investigation we considered the non-stationarity of the cathode spot as caused by its motion across the cathode surface. The motion is depicted as a sequence of steps of finite residence time $\tau_{0}$.

A discussion of the relaxation times shows that the $T_{\mathrm{cs}}(j)$ - and $T_{\mathrm{ns}}(j)$-characteristics of the stationary spot can be transferred to the moving spot. The same is not true for the $T_{\rho_{\mathrm{s}}}(j)$-characteristics.

The $T_{\mathrm{e}}$-characteristics are determined through a complicated integral Equation (28), (29). All solutions of this equation satisfy the relation $T_{\mathrm{e}}(j, I)$ $<T_{\mathrm{es}}(j, I)$.

It is noteworthy that the velocity of the spot enters into the calculation (28), (29) through the upper integral limit in the form $I / j v^{2}$. Due to this fact the dependence $v(I)$ has a decisive influence on the $T_{\mathrm{e}}$-characteristics. Considering the relation $v=c I^{v}$ the residence time $\tau_{0}$ increases with increasing current $I$ and the $T_{\mathrm{e}}$-characteristics rise monotonously with increasing current if we choose $v \leqq 1 / 2$ (case 1 ). In case 2 with $v \geqq 1 / 2$, however, the residence time $\tau_{0}$ decreases with the current and as a consequence the $T_{\mathrm{e}}$-characteristics rise only up to a certain current $\tilde{I}$ and then descend again.

In case 1 the principal structure of the E-area is not changed in comparison to the stationary state. There is still the (0)- and the (1)-mode. For all currents $I>I_{0}$ we have finite E-areas increasing with the current $I$. The critical current of the $(0)$-mode $I_{0}$ is slightly shifted to higher values in comparison to $I_{0 \mathrm{~s}}$. The critical current of the (1)-mode may be raised drastically to unphysically high values.

In case 2 we do still have a critical current $I_{0}>I_{0 \mathrm{~s}}$ for the $(0)$-mode. However, we find in comparison to the stationary E-area spectacular changes.

The (1)-mode does not exist any more ${ }^{32}$.

The most striking phenomenon, however, is the fact that now the E-area opens up at $I_{0}$, increases with increasing current $I>I_{0}$ up to a certain value $\tilde{I}$ and then starts to decrease when the current is monotonously raised beyond $I$. Finally at an upper limiting value of the current $I_{u}$ the E-area vanishes again.

In this second case therefore the spot current is limited to a finite range

$$
I_{0}<I<I_{\mathrm{u}} .
$$

We studied the dependence of the two limiting currents $\left(I_{0}, I_{1}\right)$ on the proportionality constant $(c)$ of the velocity law. The result shows that with increasing $(c)$ - that is with increasing velocity for a constant current - the interval $\Delta I=I_{\mathrm{u}}-I_{0}$ shrinks and eventually reaches the value zero. From that point on existence of the spot is precluded altogether.

A physical interpretation of these analytical results may be useful to deepen the understanding:

In the case of the stationary spot we understood that the minimum current $I_{\mathrm{cr}}$ has its physical origin in the fact that below this current the heating of the cathode is insufficient to produce the surface temperature which is necessary to provide the metal vapour and the plasma conditions which in turn yield the ion current necessary for the surface heating. For $I>I_{\text {cr }}$ there is an E-area available which increases with the current. Within this "uncertainty area" lies the poinit of solution where our physical conditions can be met.

If the spot moves the energy balance is disfavoured and consequently $T_{\mathrm{e}}$ is shifted to lower values. This cannot surprise since - contrary to the stationary spot - the moving spot after each step must built-up the surface temperature from the value $T_{0}$. In this way it will reach the stationary temperature $T_{\text {es }}$ only after an infinitely long residence time. The smaller the residence time in a step, the more will the $T_{\mathrm{e}}$-characteristic shift to smaller values, $T_{\mathrm{e}}<T_{\mathrm{es}}$.

This has an interesting consequence in connection with the dependence of the velocity on the current, $v=c I^{\prime}$. In the case where we have $v>1 / 2$ the residence time decreases with increasing current. This tends to shift the $T_{\mathrm{e}}$-characteristics with increasing current to smaller values. Depending on the parameters, this disfavouring effect may overcome the in principal favourable influence of increasing spot current on the energy balnace. In this way, we can understand the rise and the descend of the $T_{\mathrm{e}}$-characteristics in case 2. At the same time we understand the disappearance of the E-area at un upper current limit $I_{\text {u }}$.

The results described here offer themselves only to qualitative conclusions since the lack of knowledge of the velocity dependence $v(I)$ on the spot current precludes quantitative comparison:

The existence of an upper limit of the spot current $I_{\mathrm{u}}$ in case 2 of the velocity dependence not only could explain but would demand the phenomenon of spot multiplicity. If the total arc current surpasses the critical value $I_{\mathrm{u}}$ then there is no other choice for the discharge but to form additional spots 
at the cathode. The results also offer a possible explanation for the observed phenomenon of spot extinction when the spot approaches the rim of the cathodic area. Due to the influence of magnetic interaction we can expect - and experimental observation shows - that the spot velocity increases towards the rim of the cathodic discharge area. Consequently we must expect that the spot moves in the direction of increasing values of $(c)$ when it approaches the rim. The results of Fig. 8 would then predict the suicide of the spot when it reaches the critical value $\left(c_{\mathrm{cr}}\right)$ where the curves $I_{\mathrm{u}}$ and $I_{0}$ intersect.

1 G. Ecker, Betr. Plasmaphysik II, 406 [1971].

2 G. Ecker, Erg. exakt. Naturwiss. 33, 1 [1961].

3 G. Ecker, Z. Naturforsch. 26 a, 935 [1971].

${ }^{4}$ G. Ecker, Z. Naturforsch., in print [1973].

5 J. D. Cobine, and G. A. Farrall, J. Appl. Phys. 31, 2296 [1960].

6 We do not include in this analysis the more general procedure of ${ }^{4}$ but the less cumbersome and more transparent approach of ${ }^{1}$ since it will produce the essential effects.

7 H. S. Carslaw and J. C. Jaeger, Conduction of Heat in Solids, 2nd ed., Clarendon Press, Oxford 1959, p. 264.

8 Calculation of Rykalin ${ }^{9}$ and Krylovich and AbramenKO ${ }^{10}$ are not applicable here since they are based on the assumption of a constant energy influx and constant values of heat conduction and thermal diffusivity.

9 N. N. Rykalin, Thermal Basis of Welding, Academy of Science Press USSR, [1947].

10 V. L. Krylovich and T. N. Abramenko, Templofizika Vysokikh Temperature 4, 71 [1966].

11 H. S. Carslaw and J. C. Jaeger, Conduction of Heat in Solids, 2nd ed., Clarendon Press, Oxford 1959, p. 262.

12 Here and in the following, the suffix $\mathrm{s}$ designates quantities referring to the stationary solutions.

13 C. J. Gallagher and J. D. Cobine, Phys. Rev. 71, 481 [1947].

14 C. G. Smith, Phys. Rev. 73, 543 [1948].

15 S. Yamamura, J. Appl. Phys. 21, 193 [1950] •

16 C. J. Gallagher, J. Appl. Phys. 21, 798 [1950].

17 R. M. ST. John and J. G. Wienans, Phys. Rev. 94, 1097 [1954].

18 A. E. Robson and A. v. Engel, Phys. Rev. 93, 1121 [1954].

19 H. Weichel, Proceedings of the 7th International Conference on Phenomena in Ionized Gases, Beograd, Yugoslavia, 1, 749 [1966].
In summarizing we may say that our results in this paper show that the motion of the spot does not change the principal conclusions drawn in ${ }^{1}$ and ${ }^{4}$ of this treatment. It introduces however some additional features which offer a possibility to explain the phenomenon of "spot multiplicity" and "spot extinction". The results with regard to the latter two phenomena cannot be conclusive due to the insufficient knowledge of the laws of spot motion. Additional experimental and theoretical information to this latter point would be useful in many respects.

20 D. L. Murphree and R. P. Carter, Phys. Fluids 13, 1747 [1970].

21 R. L. Longini, Phys. Rev. 72, 184 [1947].

22 J. Rothstein, Phys. Rev. 78, 331 [1950].

23 A. Ware, Proc. Phys. Soc. London A 67, 869 [1954].

24 K. G. Hernquist and E. O. Johnson, Phys. Rev. 98, 1576 [1955].

25 R. M. ST. John and J. G. Wienans, Phys. Rev. 98, 1664 [1955].

26 A. Eidinger and W. Rieder, Arch. Electrotechn. 43, 94 [1957].

27 G. Ecker and K. G. Müller, Z. Naturforsch. 15 a, 577 [1958].

28 A. E. Guile, T. J. Lewis, and P. E. Secker, Proceedings of the 6th International Conference on Ionization Phenomena in Gases, Paris 2, 283 [1963].

29 H. O. SChrade, Proceedings of the 7th International Conference on Phenomena in Ionized Gases, Beograd Yugoslavia 1, 740 [1966].

30 I. G. Kesaev, Cathode Processes in the Mercury Arc, Statepower Engineering Press, Moskau 1961, p. $32-37$.

31 This cancellation of the (1)-mode at the copper cathode might raise worries about the existence of the arc in those cases - e. g. tungsten or molybdenum - where we have only the (1)-mode available. Calculations for the case of tungsten showed that here existence in the (1) -mode is possible. However, the critical minimum current $I_{1}-$ as in the case of copper - is substantially increased $I_{1}>I_{1 \mathrm{~s}}$.

32 As we already stated above, the disappearance of the (1) mode in the case of copper does not imply the same result for all materials. E.g. in the case of tungsten we have shown that for instance for a velocity law $v=I / 4[\mathrm{~cm} / \mathrm{sec} \mathrm{A}]$ existence in the (1)-mode is possible above a critical current, $I_{1}=10^{3}[\mathrm{~A}]$. 